\title{
Gestión técnica de riesgos laborales en un concesionario de vehículos
}

\section{Technical management of occupational risks in a vehicle dealer}

Cristian Laverde Albarracín

Universidad Tecnológica Equinoccial, Ecuador

Edwin Giovanny Puente Moromenacho

Universidad Internacional del Ecuador, Ecuador

Marco Vinicio Noroña Merchán

Universidad Internacional del Ecuador, Ecuador

Irene Bustillos Molina

Universidad Técnica Estatal de Quevedo, Ecuador

Autor para correspondencia: cristian.laverde@ute.edu.ec

Fecha de recepción: 23 de junio de 2018 - Fecha de aceptación: 15 de septiembre de 2018

Resumen: El presente estudio fue diseñado para determinar los factores de riesgo laboral a los cuales están expuestos los colaboradores de un concesionario de vehículos en la Ciudad de Santo Domingo en el año 2018. Los datos se obtuvieron luego de aplicar la Gestión Técnica de Riesgos Laborales a los colaboradores de la empresa, la identificación inicial de riesgos se estableció por medio de la matriz del Instituto Nacional de Seguridad e Higiene del Trabajo INSHT de España, posteriormente se procedió a la medición y evaluación de los factores de riesgos identificados como de nivel moderado e intolerables, recurriendo a los métodos internacionales específicos para determinar el nivel en el que se encuentran. Como resultado de la investigación se logró definir cuáles de los riesgos evaluados deben ser controlados y reducidos, para esto se ha planteado una propuesta de medidas de prevención y control.

Palabras Claves: riesgos laborales; prevención; identificación; evaluación; control; condiciones de trabajo

Abstract: The present study was designed to determine the occupational risk factors to which employees of a vehicle dealer are exposed in the City of Santo Domingo in 2018. The data was obtained after applying the Technical Management of Occupational Risks to the employees of the company, the initial identification of risks was established through the matrix of the National Institute of Safety and Health of Work INSHT of Spain, then proceeded to the measurement and evaluation of risk factors identified as moderate and intolerable, using specific international methods to determine the level at which they are located. As a result of the investigation it was possible to define which of the evaluated risks should be controlled and reduced, for this a proposal of prevention and control measures has been proposed.

Key words: occupational risks; prevention; identification; evaluation; control; working conditions 


\section{Introducción}

El trabajo ha sido históricamente un riesgo para la salud. Las condiciones laborales han supuesto habitualmente una amenaza a la salud que han ocasionado accidentes y enfermedades relacionadas con la salud de todo tipo. La imagen popular 1 asociada al trabajo ha sido claramente negativa. Los tiempos han cambiado de forma muy importante, pero las condiciones laborales siguen siendo preocupantes (Moreno Jiménez, 2011).

Las organizaciones, para poder sobrevivir y prosperar en un contexto dinámico donde el cambio económico y social son continuos e incluso donde la crisis económica entra en juego, necesitan tener empleados motivados y sanos, tanto física como psicológicamente; para conseguirlo, las políticas de recursos humanos deben ser consecuentes con estos valores y con un contexto tan complejo (Gómez, Hernández, Méndez 2014). La evolución de la actividad laboral ha traído consigo una mejora en la calidad de vida de los trabajadores, pero además es responsable de una serie de efectos negativos en la salud de estos (Moreira, Álvarez 2002), Acorde a todo lo anterior, las organizaciones modernas están cambiando en una dirección que se basa cada vez más en el conocimiento psicológico, la experiencia y el talento, así como en la autogestión y la atención a necesidades individuales y colectivas de los empleados, de la organización y de la sociedad en general (Salanova, Soria 2009).

Ahora bien, la salud ocupacional y la seguridad industrial conforman un binomio inseparable que garantiza la minimización de los riesgos laborales y la prevención de accidentes en el trabajo (Cortez Díaz 2002). Por riesgo laboral se entiende la probabilidad de que ocurran lesiones a las personas, daños al medio ambiente o pérdidas en los procesos y equipos dentro de un contexto laboral (Gallegos, 2012). Los accidentes laborales, en cambio, son aquellos hechos lesivos o mortales que tienen lugar durante la jornada de trabajo y que se caracterizan por ser violentos y repentinos, pero prevenibles (Cavassa, 1996). Mientras que los accidentes son evitables, los riesgos están siempre presentes y a veces sólo es posible neutralizarlos o minimizarlos a través de capacitaciones y señalizaciones que cumplen una función preventiva más que anuladora. Por tanto, puede decirse que no hay puesto de trabajo que no conlleve riesgos laborales (De la Poza 1990).

La Seguridad y Salud en el trabajo entendiéndose en un sentido amplio e integrador que englobe las prácticas tradicionales y muchas veces poco integradas de la: seguridad industrial, higiene industrial, ergonomía, psicosociología y medicina del trabajo, no ha tenido la aceptación esperable en la mayoría de las organizaciones, entre otras razones debido a, los escasos resultados demostrados por dicha actividad, lo que a su vez ha determinado que en muchas organizaciones la actividad preventiva sea relegada a un segundo plano al no considerarla parte de la productividad (Zamora, 2006)

El auge alcanzado en las últimas décadas por el sector automotriz ha propiciado la investigación y desarrollo para la mejora de las características técnicas y de diseño de los automotores, lo que ha dado paso a una mayor oferta de marcas y modelos que tratan permanentemente de reducir el impacto medioambiental de sus emisiones. Este avance es el resultado de una serie de sucesos y transformaciones que incluyen por un lado la evolución hacia la globalización del sector en el nivel internacional, así como el alineamiento a la política industrial en el nivel nacional; aspectos que le han permitido mantener un proceso de evolución 
constante (Miranda 2007). Paralelamente el negocio automotriz ha tomado forma en nuestro país con un concepto de posventa que asegura el servicio y respaldo desde el momento que un vehículo es adquirido y sale de un concesionario, teniendo la posibilidad de establecer el kilometraje y el mantenimiento que debe darse a cada vehículo, lo que ha permitido establecer cada vez mayores y mejores garantías de calidad en los vehículos.

Este nuevo enfoque de servicio ha permitido que se mejoren los estándares de calidad en el servicio luego de comprar un vehículo, pero la gran oferta de vehículos además de las medidas para desincentivar su compra han reducido los márgenes de utilidad para las empresas concesionarias, por lo que establecer resultados cualitativos y cuantitativos de la gestión en prevención de riesgos, es de vital importancia para traducir los esfuerzos financieros en mejoras de las condiciones de trabajo, en calidad y mejora en los servicios (Quiroz, Trejo 2010).

Para poder conseguir un ambiente de trabajo sano y seguro es necesaria la implementación de un sistema de gestión en seguridad y salud del trabajo, parte de este sistema incluye la gestión técnica para la cual se debe realizar la identificación, evaluación y control de los factores de riesgos propios de las actividades de las empresas, debido a que, puede decirse que no hay puesto de trabajo que no conlleve riesgos laborales (De la Poza, 1990). En el presente trabajo se presenta los resultados obtenidos de la Gestión Técnica de Riesgos Laborales, basados en la normativa legal vigente en el Ecuador aplicada a un Concesionario Automotriz.

\section{Materiales Y Métodos}

La investigación se basó en el artículo 54 de la Resolución C.D. IESS 513, en donde se manifiesta la obligatoriedad del uso de estándares y procedimientos ambientales y biológicos contenidos en normas técnicas nacionales e internacionales (Consejo Directivo IESS, 2016). Este proceso se inició con la recopilación de toda la información para definir el contexto, luego se identificaron los peligros y los riesgos (Ulloa, 2012) por cada proceso del concesionario, tanto el área operativa, como en el área administrativa, (para ello se utilizaron diagramas de flujo, listas de control), para aplicar la matriz de identificación de riesgos del Instituto de Seguridad e Higiene del Trabajo INSHT de España. En la Gestión Técnica de riesgos laborales los métodos adecuados para la evaluación de los riesgos alineados a la salud y seguridad en el trabajo, deben ser adaptados a las técnicas utilizadas en los distintos parámetros de integración, que aporten al sistema de gestión implantado (Ulloa 2012), por lo que se procedió a evaluar los riesgos laborales importantes, identificados en la matriz, con los siguientes métodos:

- William Fine (Riesgos Mecánicos)

- WBGT (Riesgo Físico)

- Medición de Ruido (Riesgo Físico)

- Comparación de Valores con tabla del Decreto Ejecutivo 2393 (Iluminación).

- Concentración de partículas (Riesgos Químicos)

- RULA (Riesgos Ergonómicos)

- Instituto Navarro de Salud Laboral (Riesgos Psicosociales)

- Messeri (Riesgo de Incendios) 
Para las diferentes mediciones, tal y como solicita la Resolución IESS CD 513, se usaron instrumentos de medición debidamente calibrados.

Población y Muestra

La población considerada en el presente trabajo fue con los 83 colaboradores del Concesionario de vehículos en estudio, dividido en las siguientes áreas:

- Administración.

- Repuestos.

- Talleres.

- Ventas.

\section{Consideraciones éticas}

El consentimiento para la realización de la presente investigación, se obtuvo por medio de la solicitud a la Gerencia General y Directorio de la empresa, pudiendo así levantar la información del total de los empleados, además de dejar constancia del anonimato y confidencialidad de los participantes, con el compromiso estricto del adecuado manejo de la información y poder establecer las condiciones reales de la empresa en el momento de la aplicación de la Gestión Técnica de Riesgos Laborales, generando oportunidades de mejora continua.

\section{Resultados Y Discusión}

\section{Riesgos Mecánicos}

Mediante William Fine se obtuvieron los siguientes resultados por el grado de peligrosidad que representan.

Los resultados muestran un riesgo en mayor medida intolerable e importante que requiere de una pronta intervención, principalmente en los talleres mecánicos y de Colisiones, causado por las herramientas que manipulan los trabajadores y a la circulación de maquinaria o vehículos en las áreas de trabajo que reducen el espacio; así también en el área administrativa se evidencia riesgo por la acumulación de archivos y cableado eléctrico que impide obstaculiza los sitios de trabajo, principalmente debajo de los escritorios, lo que impide un normal estiramiento de piernas. 
Tabla 1. Evaluación de Riesgos Mecánicos, Método de William Fine

\begin{tabular}{|c|c|c|c|}
\hline ITEM & PUESTO & FACTOR DE RIESGO & $\begin{array}{c}\text { ESTIMACIÓN } \\
\text { DEL RIESGO }\end{array}$ \\
\hline 1 & Cajera & Espacio físico reducido & \multirow{15}{*}{$\begin{array}{l}\text { RIESGO } \\
\text { INTOLERABLE }\end{array}$} \\
\hline 2 & Asesor de Repuestos & Obstáculos en el piso & \\
\hline 3 & Ayudante de enderezada & $\begin{array}{l}\text { Manejo de herramienta cortante } y / 0 \\
\text { punzante }\end{array}$ & \\
\hline 4 & Gerencia General & \multirow{2}{*}{$\begin{array}{l}\text { Desplazamiento en transporte } \\
\text { (terreste, aéreo, acuático) }\end{array}$} & \\
\hline 5 & Gerencia Comercial & & \\
\hline 6 & Técnico mecánico & \multirow{2}{*}{ Caída de objetos en manipulación } & \\
\hline 7 & Asesor de Repuestos & & \\
\hline 8 & Enderezador & $\begin{array}{l}\text { Manejo de herramienta cortante y/o } \\
\text { punzante }\end{array}$ & \\
\hline 9 & Jefe de Taller de Colisiones & $\begin{array}{l}\text { Circulación de maquinaria y vehiculos } \\
\text { en áreas de trabajo }\end{array}$ & \\
\hline 10 & Enderezador & \multirow{2}{*}{ Proyección de sólidos o líquidos } & \\
\hline 11 & Ayudante de enderezada & & \\
\hline 12 & Lavador de Vehículos & Piso irregular, resbaladizo & \\
\hline 13 & Técnico mecánico & \multirow{3}{*}{$\begin{array}{l}\text { Circulación de maquinaria y vehiculos } \\
\text { en áreas de trabajo }\end{array}$} & \\
\hline 14 & Enderezador & & \\
\hline 15 & Ayudante de enderezada & & \\
\hline 16 & Gerencia Post-Venta & \multirow{2}{*}{$\begin{array}{l}\text { Desplazamiento en transporte } \\
\text { (terreste, aéreo, acuático) }\end{array}$} & \multirow{9}{*}{$\begin{array}{c}\text { RIESGO } \\
\text { IMPORTANTE }\end{array}$} \\
\hline 17 & Vendedor externo de Repuestos & & \\
\hline 18 & Técnico de pintura & Espacio físico reducido & \\
\hline 19 & Gerencia General & Desorden & \\
\hline 20 & Logística & $\begin{array}{l}\text { Desplazamiento en transporte } \\
\text { (terreste, aéreo, acuático) }\end{array}$ & \\
\hline 21 & Técnico mecánico & \multirow{3}{*}{ Superficies o materiales calientes } & \\
\hline 22 & Enderezador & & \\
\hline 23 & Ayudante de enderezada & & \\
\hline 24 & Técnico mecánico & Trabajos de mantenimiento & \\
\hline 25 & Gerente Financiero & \multirow{5}{*}{$\begin{array}{l}\text { Desplazamiento en transporte } \\
\text { (terreste, aéreo, acuático) }\end{array}$} & \multirow{8}{*}{$\begin{array}{c}\text { RIESGO } \\
\text { MODERADO }\end{array}$} \\
\hline 26 & Jefe de Ventas & & \\
\hline 27 & Asesor Comercial & & \\
\hline 28 & Técnico mecánico & & \\
\hline 29 & Jefe de Taller de Colisiones & & \\
\hline 30 & Asesor de Repuestos & \multirow{3}{*}{$\begin{array}{l}\text { Caída de objetos por derrumbamiento } \\
0 \text { desprendimiento }\end{array}$} & \\
\hline 31 & Gerente de Recursos Humanos & & \\
\hline 32 & Asesora Comercial F\&I & & \\
\hline
\end{tabular}

\section{Riesgos Físicos}

Ruido

Tabla 2. Evaluación de Ruido Lijado - Pulido y esmerilado Actividad de Lijado y Pulido 


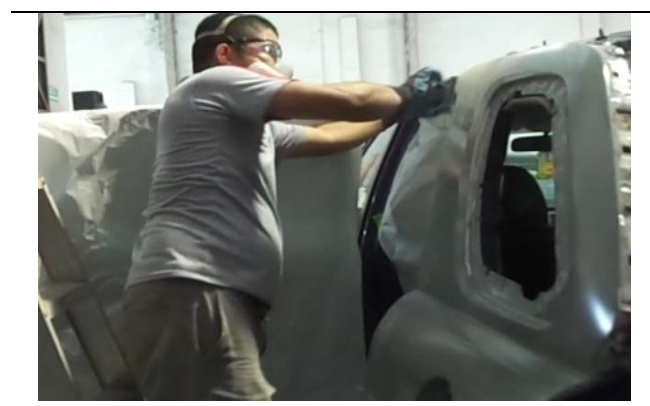

Actividad de Esmerilado:

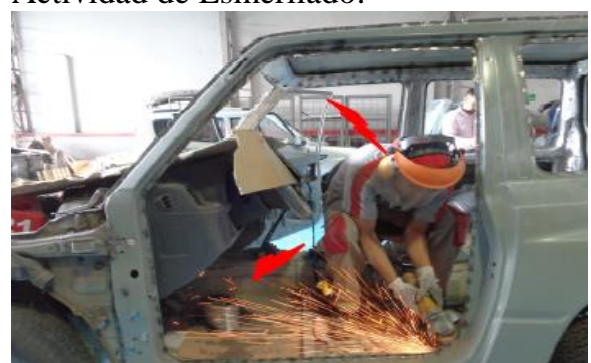

Orejera tipo diadema marca 3M código H9A.

\begin{tabular}{|ll|}
\hline EXTECH REPORT \\
LAeq d & $\mathbf{8 7 , 6 0} \mathrm{dBA}$ \\
LCeq & $93,20 \mathrm{dBC}$ \\
LZeq & $97,00 \mathrm{dBZ}$ \\
\hline
\end{tabular}

Resultados del nivel equivalente en ponderación de frecuencia A, C y Z

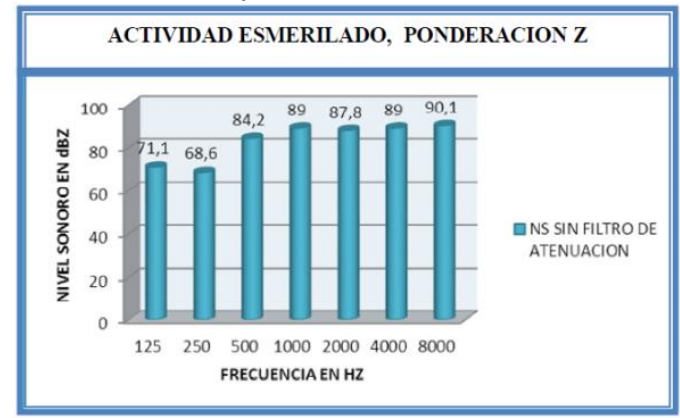

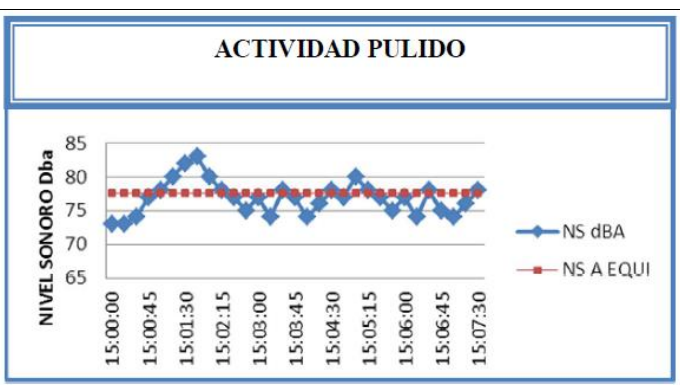
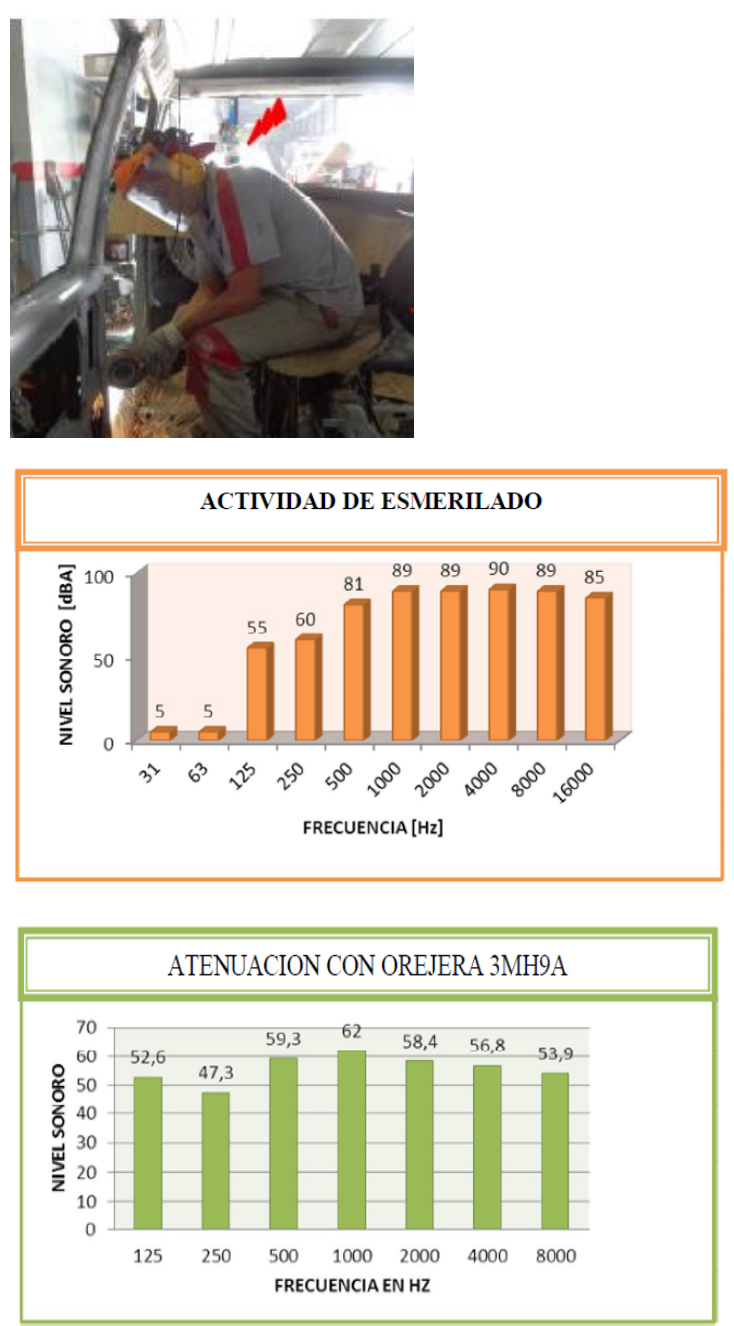

Tabla 3. Evaluación de lavado de carros - pintado y lacado

Lavador de Carros 


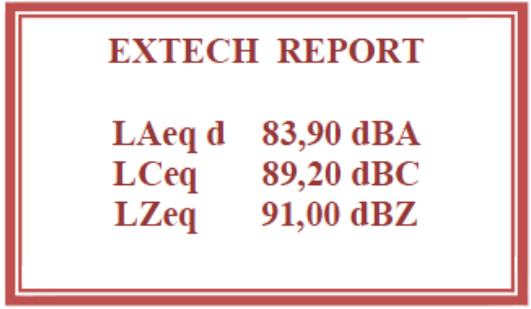

Resultados del nivel equivalente en ponderación de frecuencia A, C y Z

Actividad de Pintado y Lacado
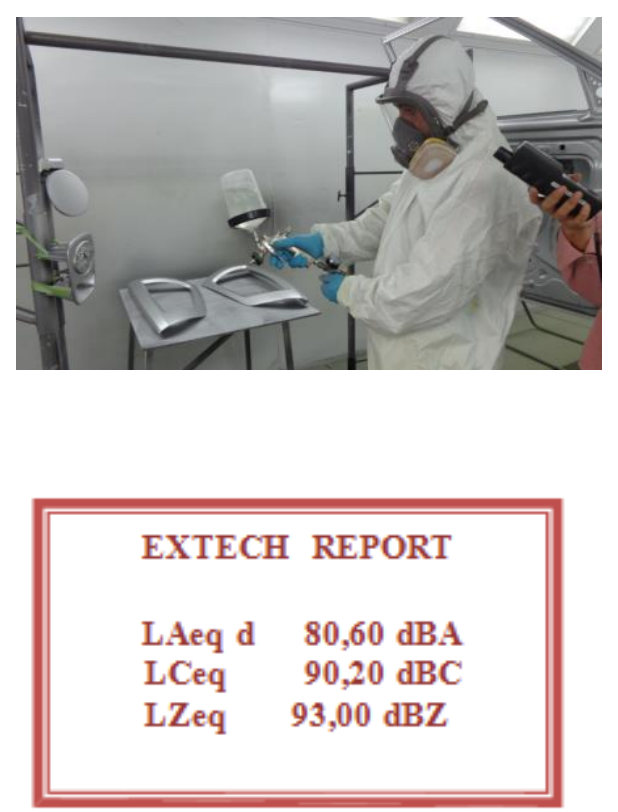

Resultados del nivel equivalente en ponderación de frecuencia A, C y Z
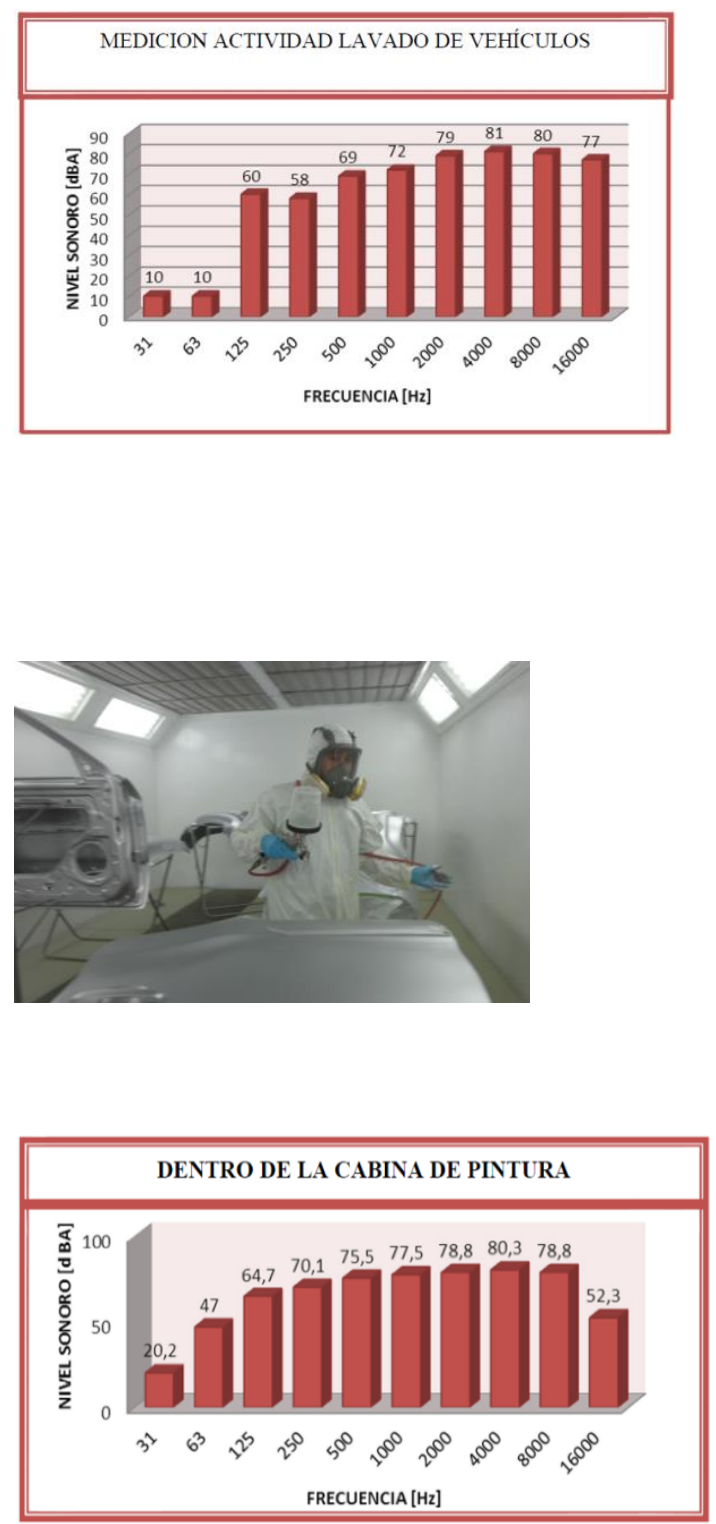

Tabla 4. Evaluación de ruido por uso de mazo y martillo - cambio de llantas Uso de mazo y martillo 

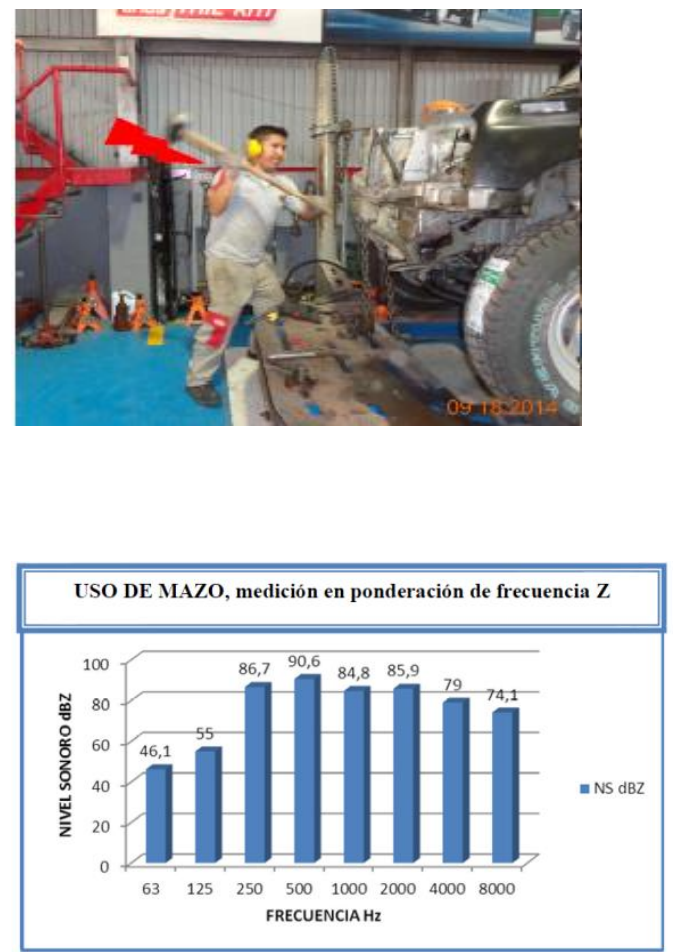

Sin filtro y la frecuencia (banda de octava)

Cambio de Llantas

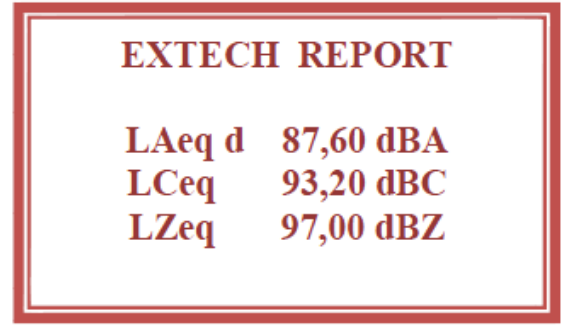

Resultados del nivel equivalente en ponderación de frecuencia A, C y Z

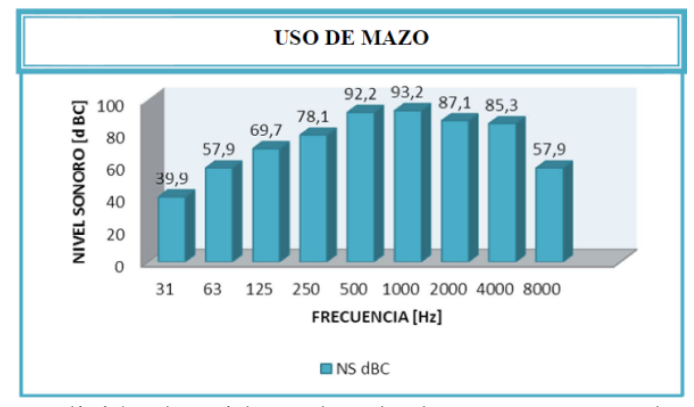

Medición de ruido en banda de octava en ponderación $\mathrm{C}$ (impulso)

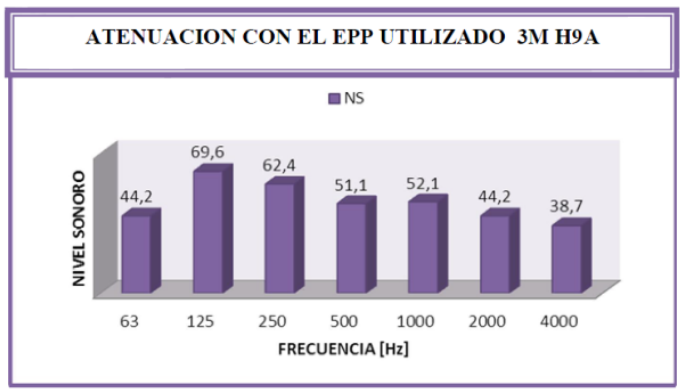

MEDICION DE ACTIVIDAD CAMBIO DE LLANTAS

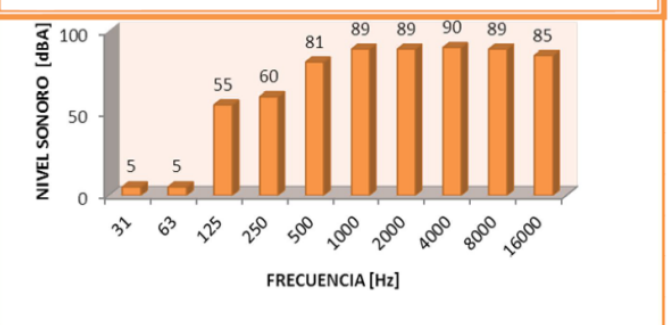

\section{Temperatura / Estrés térmico}

En las áreas donde se realizó la medición por los resultados obtenidos NO existe problema de estrés térmico. Estos valores cambian dependiendo de la hora y el clima de la ciudad. 
Tabla 5. Condiciones de confort higrotérmico

\begin{tabular}{|c|c|c|c|c|}
\hline ÁREA & HORA & ACTIVIDAD & MEDICIÓN WBGT $^{\circ}$ & OBSERVACIONES \\
\hline \multirow[t]{3}{*}{$\begin{array}{l}\text { Cabina } \\
\text { de } \\
\text { pintura }\end{array}$} & $15: 30 \quad 15: 40$ & $\begin{array}{l}\text { Área caliente y húmeda. } \\
\text { Se tiene sistema ventilación y } \\
\text { extracción. }\end{array}$ & $23^{\circ}$ & $\begin{array}{l}\text { EPP'S: protección } \\
\text { respiratoria, guantes, botín } \\
\text { con punta de acero. }\end{array}$ \\
\hline & $16: 0016: 10$ & $\begin{array}{l}\text { Cabina baja, área } \\
\text { limitada. } \\
\text { Trabajo bajo techo. }\end{array}$ & $23,7^{\circ}$ & Postura: Trabajo de pie \\
\hline & & $\begin{array}{l}\text { TRABAJO FISICO LIGERO } \\
\text { movimiento de manos y } \\
\text { cuerpo, No hay levantamiento } \\
\text { de carga. }\end{array}$ & & $\begin{array}{l}\text { Trabajo físico: ligero. } \\
\text { Ambiente homogéneo. } \\
\text { Medición en la cintura de los } \\
\text { trabajadores }\end{array}$ \\
\hline
\end{tabular}

Hidratación al personal en lugares cercanos a la actividad que realizas, por la zona climática y la actividad, se sugiere hidratación continua del personal, se les debe indicar que deben hacerla.

\section{Iluminación}

Los resultados muestran que la iluminación no es la correcta para los puestos de trabajo del Jefe de Ventas, Asistente Contable 2 y en Bodega tanto en el sitio de trabajo como entre las estanterías, este contraste se hizo con lo establecido en el Decreto Ejecutivo 2393 (Laborales, 2004)

Tabla 6. Mediciones de Iluminación

\begin{tabular}{|c|c|c|c|}
\hline PUESTO & \multicolumn{2}{|c|}{ LUZ (Ix) } & $\begin{array}{c}\text { TIPO DE } \\
\text { LUMINARIA }\end{array}$ \\
\hline \multicolumn{4}{|l|}{ VENTAS } \\
\hline Jefe de ventas & O & 110 & Fluorescente \\
\hline Asesor & O & 310 & Fluorescente \\
\hline Post Ventas & 0 & 521 & Fluorescente \\
\hline \multicolumn{4}{|l|}{ ADMINISTRACION } \\
\hline Asistente contable 1 & 0 & 1250 & Fluorescente \\
\hline Asistente contable 2 & 0 & 150 & Fluorescente \\
\hline Cajera & 0 & 750 & Fluorescente \\
\hline Recepcionista - Asistente & 0 & 450 & Fluorescente \\
\hline $\begin{array}{l}\text { Bodega } \\
\text { * En el escritorio } \\
\text { * Entre las estanterías } \\
\text { * Entre las estanterías }\end{array}$ & $\begin{array}{l}0 \\
0 \\
0\end{array}$ & $\begin{array}{c}150 \\
65 \\
175 \\
\end{array}$ & $\begin{array}{l}\text { Fluorescente } \\
\text { Fluorescente } \\
\text { Fluorescente }\end{array}$ \\
\hline
\end{tabular}

\section{Riesgos Químicos}

El sistema de extracción desfoga dentro del taller, hacia la puerta de acceso posterior. La disipación es rápida, en ese lugar no es de trabajo sino de tránsito de los trabajadores, pero llega a molestar en las áreas cercanas. 
Masillado

Valor máximo: $\quad 8,742 \mathrm{Mg} / \mathrm{M} 3$

Valor promedio: 2,75 Mg/M3 Tiempo de medición: 5 Minutos (Ref.: Partícula Respirable).

Lijado y pulido

\section{MEDICION EN LIJADO Y PULIDO}

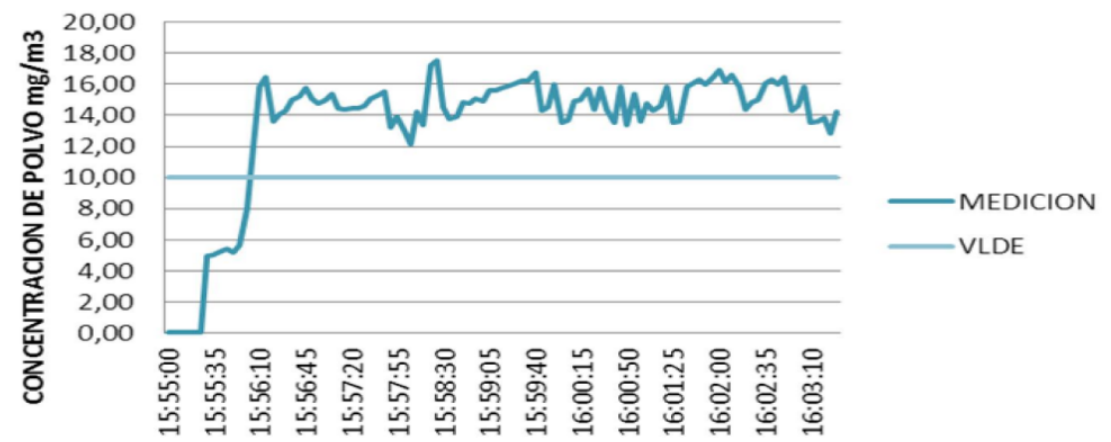

Valor máximo: $17,46 \mathrm{mg} / \mathrm{m} 3$

Valor promedio: 13,87 mg/m3 Tiempo de medición: 8 minutos

Esmerilado y soldadura
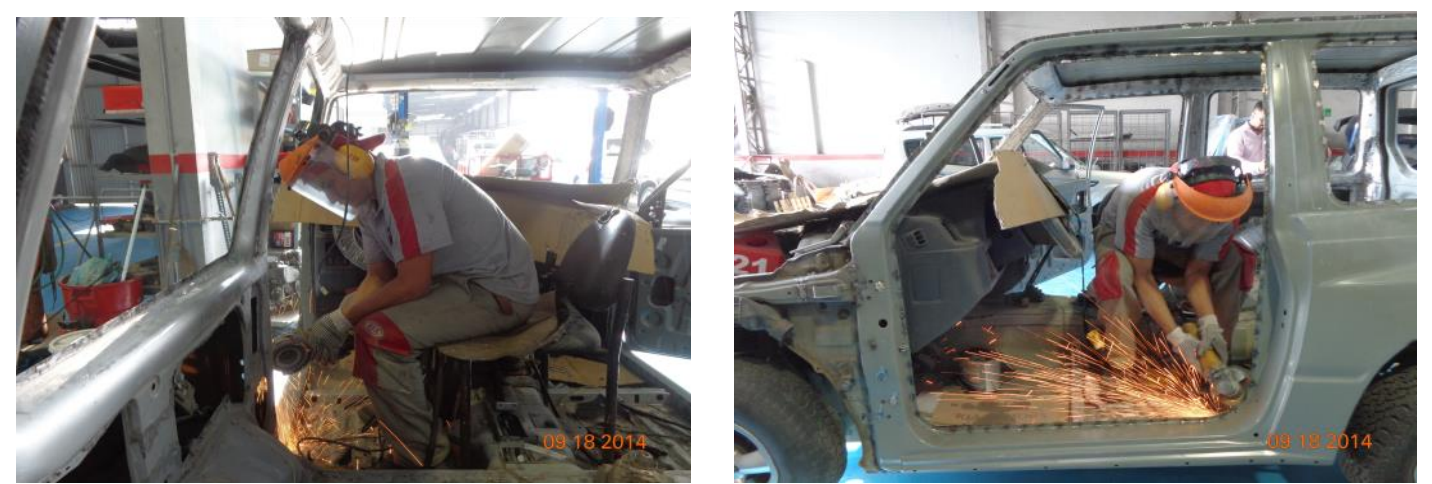

Valor máximo: $13,46 \mathrm{mg} / \mathrm{m} 3$

Valor promedio: 5,634 mg/m3 Tiempo de medición: 5 minutos

Área de la cabina de pintura 


\section{MEDICION EN LA CABINA DE PINTURA}

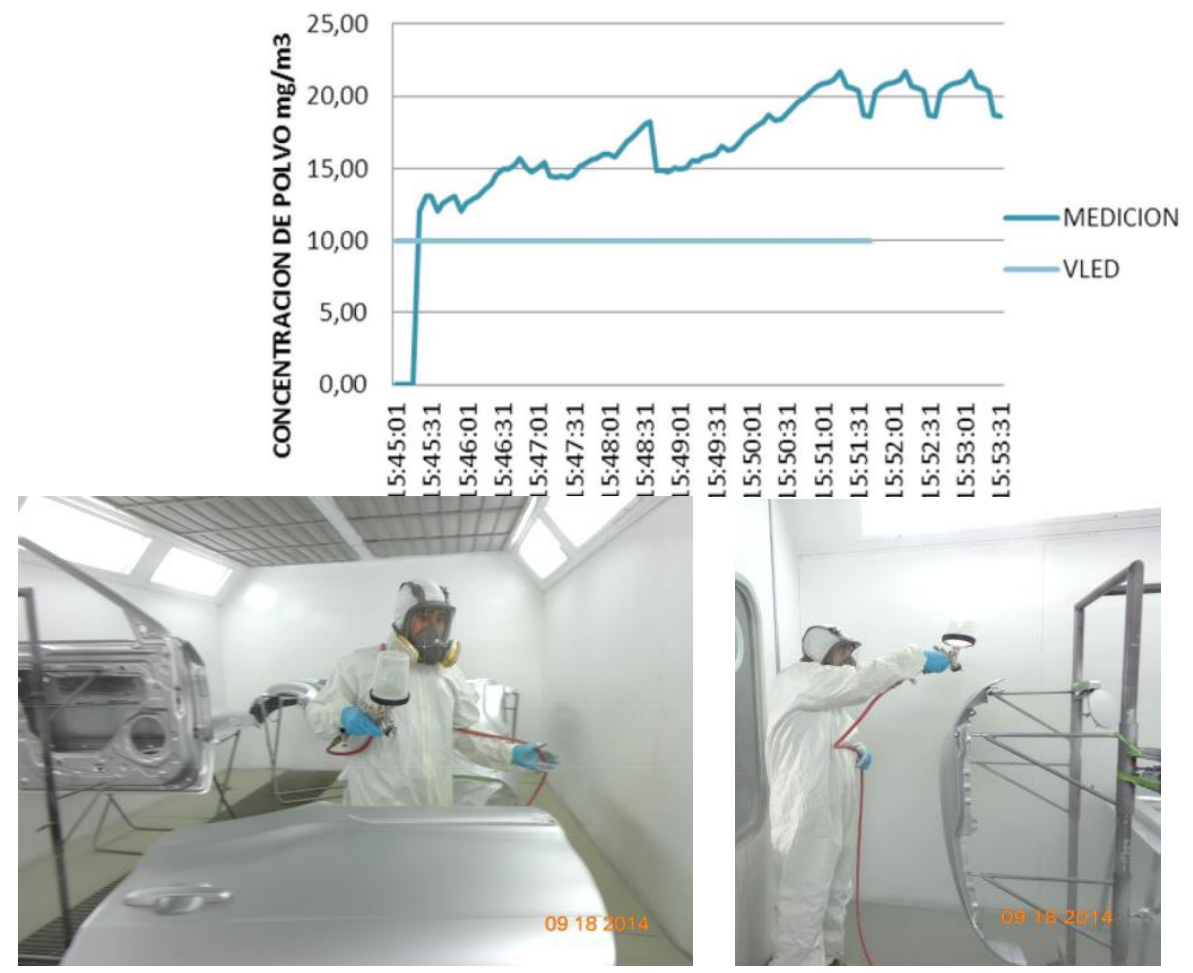

Valor máximo: $\quad 21,46 \mathrm{mg} / \mathrm{m} 3$

Valor promedio: $16,671 \mathrm{mg} / \mathrm{m} 3$ Tiempo de medición: 8 minutos

Extractor de la cabina de pintura

\section{SECTOR DEL EXTRACTOR DE LA} CABINA DE PINTURA
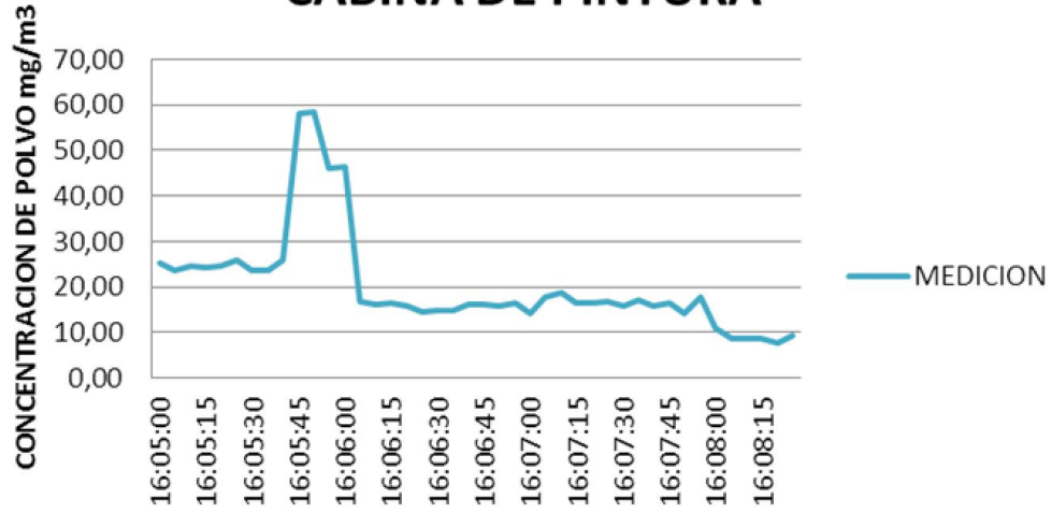

Valor máximo: $\quad 58,46 \mathrm{mg} / \mathrm{m} 3$

Valor promedio: 17,50 mg/m3 Tiempo de medición: 3 minutos

Esta concentración es cada vez que funciona el sistema extractor de la cabina. 


\section{Riesgos Psicosociales}

Se empleó el Método del Instituto Navarro de Salud Laboral (INSL), a los 82 colaboradores.

Tabla 8. Evaluación de Riesgos Psicosociales, Método INSL

\begin{tabular}{lll}
\hline FACTOR EVALUADO & $\%$ & CALIFICACIÓN \\
\hline Participación Implicación y Responsabilidad & $57 \%$ & INADECUADO \\
Formación, Información y Comunicación & $43 \%$ & INADECUADO \\
Gestión Del Tiempo & $61 \%$ & MUY INADECUADO \\
Cohesión De Grupo & $41 \%$ & INADECUADO \\
Mobbing Acoso Laboral & $6 \%$ & NORMAL
\end{tabular}

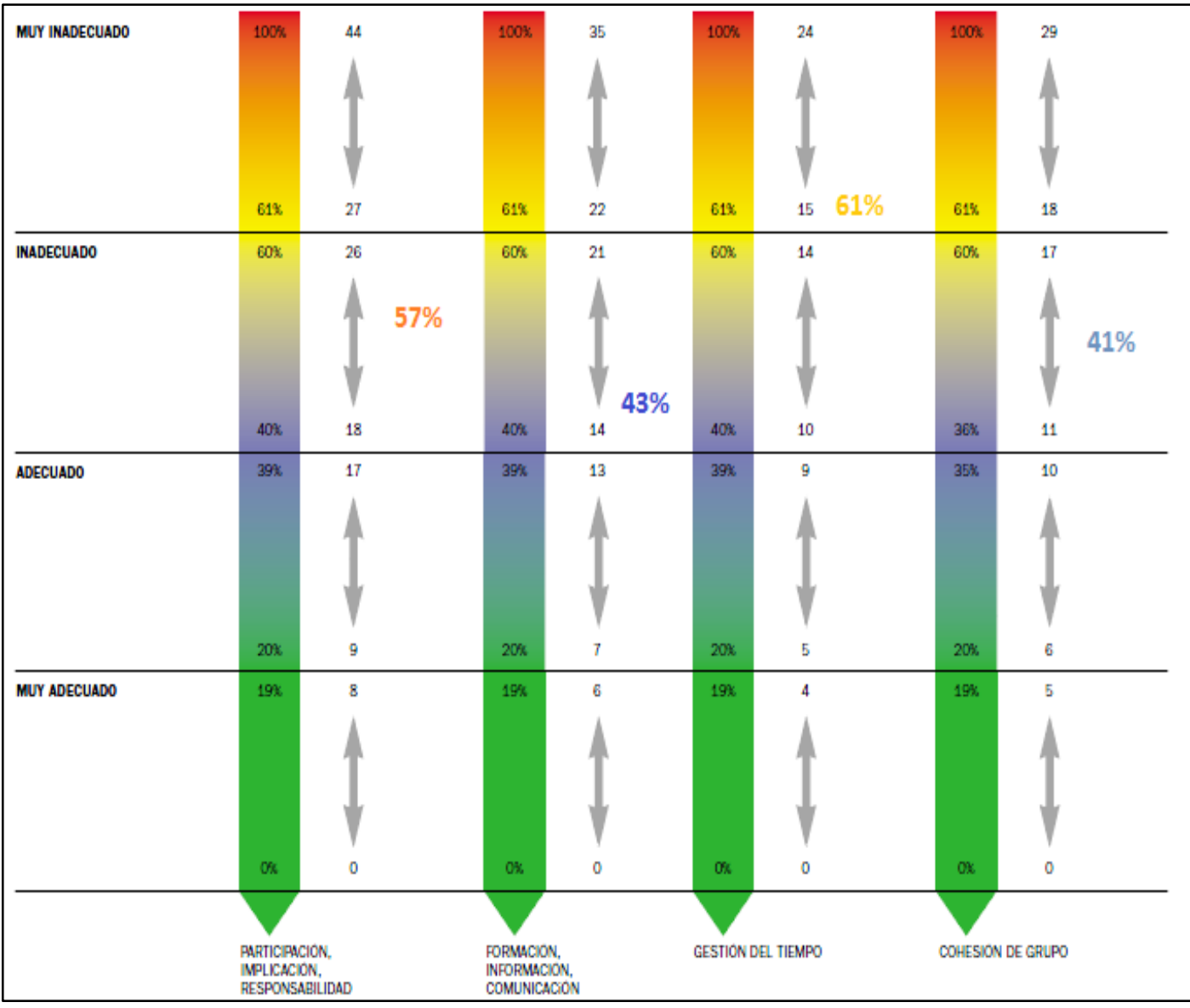

Figura 1. Evaluación de Riesgos Psicosociales INSL

La componente psicosocial, presenta un riesgo importante, por lo que el control debe tener intervención inmediata.

Riesgos Ergonómicos 
Se utilizó el método RULA para esta evaluación de riesgos ergonómicos, provocados por las diferentes posturas de trabajo, movimientos repetitivos y levantamiento de cargas, dando como resultado los porcentajes siguientes

Tabla 9. Evaluación de Riesgos Ergonómicos por el método RULA

\begin{tabular}{|c|c|c|c|c|c|c|c|c|c|c|c|}
\hline \multirow[b]{2}{*}{ PUESTO } & \multicolumn{5}{|c|}{ GRUPO A } & \multicolumn{4}{|c|}{ GRUPO B } & \multirow{2}{*}{ 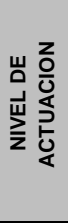 } & \multirow[b]{2}{*}{ OBSERVACION } \\
\hline & 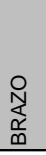 & 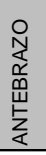 & 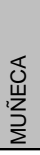 & 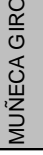 & $\begin{array}{l}z \\
\frac{2}{0} \\
\frac{1}{2} \\
\frac{1}{2} \\
\text { a } \\
\end{array}$ & $\begin{array}{l}\text { 올 } \\
\text { 岂 }\end{array}$ & 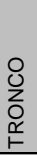 & $\frac{\omega}{\alpha}$ & $\begin{array}{l}z \\
\frac{0}{0} \\
\frac{1}{2} \\
\frac{5}{3} \\
0 \\
\end{array}$ & & \\
\hline Administrativo & 2 & 2 & 2 & 1 & 3 & 3 & 1 & 2 & 3 & 2 & $\begin{array}{l}\text { Se requiere más investigación y } \\
\text { se pueden requerir cambios }\end{array}$ \\
\hline Cambio de llantas & 5 & 2 & 1 & 1 & 5 & 3 & 5 & 2 & 7 & 4 & $\begin{array}{l}\text { Se requiere realizar } \\
\text { inmediatamente actividades de } \\
\text { investigación y cambios en la } \\
\text { tarea }\end{array}$ \\
\hline Esmerilado & 6 & 3 & 2 & 1 & 9 & 5 & 6 & 2 & 8 & 4 & $\begin{array}{l}\text { Se requiere realizar } \\
\text { inmediatamente actividades de } \\
\text { investigación y cambios en la } \\
\text { tarea }\end{array}$ \\
\hline Lavador de carros & 5 & 3 & 2 & 1 & 6 & 3 & 5 & 2 & 7 & 4 & $\begin{array}{l}\text { Se requiere realizar } \\
\text { inmediatamente actividades de } \\
\text { investigación y cambios en la } \\
\text { tarea }\end{array}$ \\
\hline Lijado y Pulido & 3 & 6 & 2 & 1 & 9 & 3 & 5 & 1 & 6 & 4 & $\begin{array}{l}\text { Se requiere realizar } \\
\text { inmediatamente actividades de } \\
\text { investigación y cambios en la } \\
\text { tarea }\end{array}$ \\
\hline $\begin{array}{l}\text { Pintura - Lacado } \\
\text { dentro de la cabina }\end{array}$ & 5 & 3 & 2 & 1 & 6 & 3 & 6 & 2 & 7 & 4 & $\begin{array}{l}\text { Se requiere realizar } \\
\text { inmediatamente actividades de } \\
\text { investigación y cambios en la } \\
\text { tarea }\end{array}$ \\
\hline $\begin{array}{l}\text { Reparación de } \\
\text { motor }\end{array}$ & 5 & 3 & 1 & 1 & 6 & 3 & 6 & 2 & 7 & 4 & $\begin{array}{l}\text { Se requiere realizar } \\
\text { inmediatamente actividades de } \\
\text { investigación y cambios en la } \\
\text { tarea }\end{array}$ \\
\hline Uso de mazo & 5 & 3 & 2 & 2 & 7 & 3 & 5 & 2 & 7 & 4 & $\begin{array}{l}\text { Se requiere realizar } \\
\text { inmediatamente actividades de } \\
\text { investigación y cambios en la } \\
\text { tarea }\end{array}$ \\
\hline Cambio de aceite & 5 & 3 & 3 & 1 & 7 & 4 & 3 & 2 & 7 & 4 & $\begin{array}{l}\text { Se requiere realizar } \\
\text { inmediatamente actividades de } \\
\text { investigación y cambios en la } \\
\text { tarea }\end{array}$ \\
\hline
\end{tabular}

\section{Riesgo De Incendios}

Se presenta riesgo medio, por lo que las medidas de intervención son controlables.

Tabla 10Evaluación de Riesgo Incendios Método Messeri

\begin{tabular}{lll}
\hline ÁREA & VALOR DEL RIESGO & CALIFICACION DE RIESGO \\
\hline Oficinas Centro de Colisiones & 5.16 & Riesgo Medio \\
Bodega de repuestos & 5.53 & Riesgo Medio \\
Oficinas Administración y Ventas & 5.86 & Riesgo Medio \\
Taller Mecánico & 5.9 & Riesgo Medio \\
Taller de Colisiones & 4.87 & Riesgo Medio \\
\hline
\end{tabular}




\section{Conclusiones Y Recomendaciones}

Aplicada la Gestión Técnica de Riesgos Laborales, es necesario llevar acciones específicas a fin de controlar y disminuir aquellos riesgos que intolerables e importantes que pueden acarrear serios problemas, para lo cual se ha planteado la siguiente propuesta de medidas de prevención y control.

\section{Acciones específicas}

- Programa de capacitación y formación en los temas necesarios en seguridad y salud ocupacional de acuerdo con las actividades que se realizan en la empresa y a los factores de riesgos presente.

- Programa de mantenimiento.

- Programa Integral de Seguridad y Salud en el Trabajo.

Medidas para la prevención de riesgos físicos

- Programa de mantenimiento a las máquinas y equipos de trabajo.

- Retirar las oficinas administrativas del interior del taller de colisiones, para mejorar la iluminación y disminuir el ruido para el personal que no realiza actividades operativas.

- Instalar luminarias sobre los sitios de trabajo.

- Establecer un cronograma de capacitación en uso y mantenimiento del equipo de protección personal.

\section{Medidas para la prevención de riesgos mecánicos}

- El sistema de extracción existente en la cabina de pintura debe prolongarse para que desfogue fuera del taller de colisiones.

- Inducción al personal que trabaja en el área del centro de colisiones o que puedan circular por las mismas, sobre las características y peligros de estos contaminantes. Colocar señales o carteles de advertencia de que es un área en donde existe presencia de vapores orgánicos.

Medidas para la prevención de riesgos químicos.

- Protección colectiva, se debe mejorar la calidad del aire con sistemas de circulación de aire, extractores y ventiladores.

- Mantenimiento preventivo de los conductos y canales de ventilación para asegurar una mejora en la calidad del aire.

- Instalación de sistemas de extracción dirigida a la tarea específica.

- Para el caso de la cabina de pintura del centro de colisiones, tomar medidas administrativas para bajar el tiempo de permanencia en el medio contaminante.

- Realizar mediciones continuas sobre la concentración de contaminantes.

- Procedimiento por seguir para la preparación de la pintura, una vez utilizado el químico se debe tapar y ubicarlo en el sitio correspondiente para evitar la contaminación del ambiente y derrames de este. 
- Obligatoriedad de usar los equipos de protección individual en el momento de la preparación de la pintura (respirador de media cara con filtro químico para vapores orgánicos, gafas, traje desechable y guantes).

\section{Medida para prevención de riesgos ergonómicos}

- Dotar de medios auxiliares como elevadores o coches para el transporte de objetos o piezas de vehículos, especialmente si son pesadas o voluminosas.

- Establecer un procedimiento a seguir para el manejo de cargas, en donde se deberá indicar que se observe la existencia de bordes cortantes o temperatura a la que se encuentran las partes y piezas,

- Sujetar o anclar firmemente las estanterías a elementos sólidos, tales como paredes o pisos, evitar la sobrecarga de estanterías y zonas de almacenamiento, los materiales almacenados no deben sobresalir y colocar los objetos más pesados en la zona inferior de las estanterías.

- Señalizar los lugares en donde sobresalgan objetos, máquinas o estructuras inmóviles.

- Mantener las vías de tránsito despejadas y eliminar cosas innecesarias.

- Capacitación en postura correcta frente a una pantalla de visualización digital, descansos obligatorios y cambio de actividad para evitar problemas músculo esqueléticos y posibles complicaciones con estrés debido a jornadas muy largas de trabajo.

- Adecuar los computadores utilizando equipos fijos y tomando en cuenta que los ojos deben quedar a la misma altura que la parte superior de la pantalla y, que la distancia entre la pantalla y el rostro del operador debe estar alrededor de 45 a $91 \mathrm{~cm}$, con el fin de evitar la inclinación hacia la pantalla.

- Dotar de unas sillas con respaldo lumbar, regulable en altura y con descansabrazos.

\section{Medidas para la prevención de riesgos psicosociales}

- Hacer un día de esparcimiento cada 3 meses con capacitaciones en cómo evitar el estrés laboral, trabajo en equipo.

- Implementación tiempos de recuperación (pausas activas) por cada hora uno con duración mínima de 5 minutos.

- Establecer un sistema de comunicación eficaz que abarque tanto personal de línea, supervisores y jefaturas en todos los temas de interés en la empresa.

- Capacitar a los supervisores y jefes de área en temas de comunicación efectiva, resolución de conflictos, coaching.

- Realizar el reconocimiento de los logros alcanzados por los trabajadores en cuanto a productividad.

- Favorecer el manejo de habilidades y la oportunidad de aprendizaje a través del trabajo.

\section{Medidas para la prevención de riesgo de incendio}

- Cambio del sistema de detección de humo

- Realizar inspecciones periódicas a las condiciones de almacenamiento en bodega, mantenimiento, oficinas, sistema eléctrico y sistema de detección de incendios en todas las instalaciones de la empresa. 
- Mantener a las brigadas de incendios, evacuación, primeros auxilios y comunicación con instrucción periódica en el manejo adecuado de extintores, plan de emergencia de la empresa.

\section{Bibliografía}

Cavassa, C. R. (1996). Seguridad industrial: un enfoque integral. Editorial Limusa.

Consejo Directivo, I. E. S. S. (2016). Reglamento al Seguro General de Riesgos del Trabajo.

Cortés Díaz, J. M. (2002). Seguridad e higiene del trabajo: técnicas de prevención de riesgos laborales.

De la Poza Lleida, J. M. (1990). Seguridad e higiene profesional: con las normas comunitarias europeas y norteamericanas. Paraninfo.

Gallegos, W. L. A. (2012). Revisión histórica de la salud ocupacional y la seguridad industrial industrial. Revista cubana de salud y trabajo, 13(3), 45-52.

Gómez Rojas, P., Hernández Guerrero, J., \& Méndez Campos, M. D. (2014). Factores de riesgo psicosocial y satisfacción laboral en una empresa chilena del área de la minería. Ciencia \& trabajo, 16(49), 9-16.

Laborales, M. D. (2004). Reglamento de Seguridad y Salud de los Trabajadores y Mejoramiento del Medio Ambiente de Trabajo. Decreto Ejecutivo (2393).

Miranda, A. V. (2007). La industria automotriz en México: Antecedentes, situación actual y perspectivas. Contaduría y administración, (221), 209-246.

Moreira, J. M., \& Álvarez, M. C. (2002). Clima organizacional y estrés en una unidad de alto riesgo. Emergencias, 14(1), 6-12.

Moreno Jiménez, B. (2011). Factores y riesgos laborales psicosociales: conceptualización, historia y cambios actuales. Medicina y Seguridad del trabajo, 57, 4-19.

Quiroz Trejo, J. O. (2010). Taylorismo, fordismo y la administración científica en la industria automotriz.

Salanova Soria, M. (2009). Organizaciones saludables, organizaciones resilientes.

Ulloa-Enríquez, M. Á. (2012). Riesgos del Trabajo en el Sistema de Gestión de Calidad. Ingeniería Industrial, 33(2), 100-111.

Zamora, L. V. (2006). Gestión Integral e Integrada de Seguridad y Salud Modelo Ecuador II. 